UCSBTH-92-17

YCTP-92-P37

hep-th/9210012

\title{
Black Holes Coupled to a Massive Dilaton
}

\author{
JAMES H. HORNE ${ }^{\dagger}$ \\ Department of Physics \\ Yale University \\ New Haven, CT 06511-8167 \\ Gary T. Horowitz* \\ Department of Physics \\ University of California \\ Santa Barbara, CA 93106-9530
}

\begin{abstract}
We investigate charged black holes coupled to a massive dilaton. It is shown that black holes which are large compared to the Compton wavelength of the dilaton resemble the Reissner-Nordström solution, while those which are smaller than this scale resemble the massless dilaton solutions. Black holes of order the Compton wavelength of the dilaton can have wormholes outside the event horizon in the string metric. Unlike all previous black hole solutions, nearly extremal and extremal black holes (of any size) repel each other. We argue that extremal black holes are quantum mechanically unstable to decay into several widely separated black holes. We present analytic arguments and extensive numerical results to support these conclusions.
\end{abstract}

$10 / 92$

$\dagger$ Email address: jhh@waldzell.physics.yale.edu

* Email address: gary@cosmic.physics.ucsb.edu 


\section{Introduction}

There has been increasing interest in the properties of black holes in string theory. One of the original motivations was the desire to gain a better understanding of the allowed causal structure and possible singularities in this theory. However, it has since been realized that modifications arising from string theory may help to resolve some of the puzzles associated with black hole evaporation [1]. Although an exact black hole solution in string theory is now known in two spacetime dimensions [2], we wish to consider the more physical case of four dimensions where the exact black hole solution is not known. We can however work with the low energy string equations of motion. Solutions to these equations should be close to an exact string solution whenever the curvature is small compared to the Planck scale.

Solutions to the low energy string equations of motion describing black holes are known [3] 四, and differ from the standard Reissner-Nordström solution of general relativity when the charge is nonzero. The reason for this difference is the existence of a scalar field called the dilaton. Although the difference is negligible outside the event horizon when the ratio of the charge to the mass is small, it becomes significant near the extremal limit. In particular, in terms of the metric that strings couple directly to, an extremal magnetically charged black hole has neither a curvature singularity nor a horizon. In its place is an infinite throat. The fact that a potentially infinite volume of space may be present inside an object that appears small to an observer outside the throat has been conjectured to play a role in resolving the information problem that arises when black holes evaporate [5].

Unfortunately, there is a serious omission in the investigations that have been performed so far. In low energy string theory, the dilaton is massless. But a truly massless dilaton violates the equivalence principle and is inconsistent with observations [6]. Since the dilaton is in the same supermultiplet as the graviton, it cannot get a mass while maintaining supersymmetry. However supersymmetry is certainly broken at low energies, and it is widely believed (and hoped!) that when this occurs, the dilaton will become massive. Thus physically the most interesting case is black holes coupled to a massive dilaton. This is the subject of the present investigation.

We will show that for large black holes, the spacetime resembles the ReissnerNordström solution. When the charge is comparable to the mass, there is both an outer (event) horizon and an inner (Cauchy) horizon. In the extremal limit, these two horizons coincide. For small black holes, the solution resembles the massless dilaton case. There is 
only a single horizon, and in the extremal limit, it moves off to infinity leaving an infinite throat. So infinite throats exist even in the more realistic theory with a massive dilaton. The scale at which the black hole changes character is simply the inverse mass, or Compton wavelength, of the dilaton. This is physically reasonable. When the Schwarzschild radius is less than the Compton wavelength, the black hole does not see the mass, and behaves like the massless case. When the Schwarzschild radius is much larger, the dilaton is essentially fixed at its minimum value and does not change enough to modify the geometry. Numerically, if the dilaton has a mass of $1 \mathrm{TeV}$, the transition occurs at a black hole mass of approximately $10^{11}$ gms. So the dilaton does not affect solar mass sized black holes, but could play an important role in the evaporation of smaller holes.

Charged black holes with a massless dilaton (and $\phi \rightarrow 0$ at infinity) only exist when $Q^{2} \leq 2 M^{2}$. This upper limit on the charge for a given mass is larger than the usual bound $Q^{2}=M^{2}$ without the dilaton. We will see that black holes with a massive dilaton have a maximal charge to mass ratio which depends on the size of the black hole. It appears to vary continuously from $Q^{2} / M^{2}=2$ for small black holes to $Q^{2} / M^{2}=1$ for large ones. The fact that the charge can be greater than the mass has the following important consequence. Two widely separated Reissner-Nordström black holes will attract each other unless $Q=M$. Since this is the extremal limit, it is not possible for black holes to be repulsive. When a massless dilaton is added, it adds an extra attractive force. So even though the extremal limit has a larger charge to mass ratio, this limit again corresponds to the point where the forces cancel at large separations. With a massive dilaton, the situation is different. We will see that at large distances, the solution always approaches the Reissner-Nordström metric, and the force associated with the dilaton is negligible. Since the presence of the dilaton near the horizon allows $Q^{2}>M^{2}$, one has black holes which (at large separations) repel each other! To the best of our knowledge, these are the first examples of gravitationally bound objects which are repulsive. Since $Q=M$ is not the extremal limit, initially static black holes will Hawking radiate and start to repel each other. We will argue that one consequence of this repulsive force is that large extremal black holes are quantum mechanically unstable to decay into multiple extremal black holes.

Another unusual property of black holes coupled to a massive dilaton is the presence of wormholes.1 1 It is well known that the maximally extended Schwarzschild solution contains

1 We will use the word "wormhole" to describe a region of space where the area of spheres decreases and then increases again. These are three dimensional - not four dimensional - objects. See [0] for another example of this type of wormhole. 
a wormhole in the sense that a spacelike surface connecting the two asymptotically flat regions reaches a minimum size inside (or on) the event horizon. This wormhole, however, cannot be transversed by physical observers since it quickly collapses to zero size. We will see that the string metrics describing certain black holes with a massive dilaton have wormholes outside the event horizon. These wormholes are static and can be transversed by strings falling into the black holes.

The exact form of the dilaton potential in string theory is not known. We will consider the simplest choice $m^{2} \phi^{2}$ and later briefly discuss what effect more realistic choices would have. Of course, for a general potential, when $\phi$ is small and near its minimum, $m^{2} \phi^{2}$ is a good approximation. Adding this term to the standard low energy string action yields

$$
S=\int d^{4} x\left[\mathcal{R}-2(\nabla \phi)^{2}-2 m^{2} \phi^{2}-e^{-2 \phi} F^{2}\right]
$$

where $\mathcal{R}$ is the scalar curvature and $F_{\mu \nu}$ is the Maxwell field strength. As indicated in (1.1), we will work with the metric having the standard Einstein action. This is related to the metric that the strings couple directly to via the conformal rescaling $\hat{g}_{\mu \nu}=e^{2 \phi} g_{\mu \nu}$. Both metrics have the same causal structure except in the case of extremal magnetically charged black holes, where a singular horizon in the Einstein metric corresponds to an infinite throat in the string metric. The equations of motion of (1.1) are

$$
\begin{aligned}
0 & =\nabla_{\mu}\left(e^{-2 \phi} F^{\mu \nu}\right), \\
0 & =\nabla^{2} \phi-m^{2} \phi+\frac{1}{2} e^{-2 \phi} F^{2}, \\
\mathcal{R}_{\mu \nu} & =2 \nabla_{\mu} \phi \nabla_{\nu} \phi+2 e^{-2 \phi} F_{\mu \rho} F_{\nu}{ }^{\rho}+g_{\mu \nu}\left[m^{2} \phi^{2}-\frac{1}{2} e^{-2 \phi} F^{2}\right] .
\end{aligned}
$$

These equations are invariant under the same electromagnetic duality transformation that holds when $m=0$. Namely, one can replace $F_{\mu \nu}$ and $\phi$ with $\tilde{F}_{\mu \nu} \equiv \frac{1}{2} e^{-2 \phi} \epsilon_{\mu \nu}{ }^{\lambda \rho} F_{\lambda \rho}$ and $\tilde{\phi}=-\phi$. We will consider a purely magnetically charged black hole: $F=Q \sin \theta d \theta \wedge d \varphi$. Electrically charged holes can be obtained by applying the above duality.

We are interested in static, spherically symmetric solutions. Unfortunately, we do not expect that exact solutions can be expressed in a simple closed form. We will study these solutions using two different forms of the metric. We will first choose coordinates so that the metric takes the form

$$
d s^{2}=-\lambda d t^{2}+\frac{d r^{2}}{\lambda}+R^{2} d \Omega
$$

where $\lambda$ and $R$ are functions of $r$ only. This has the advantage that both the massless dilaton black holes and no dilaton black holes (i.e. the Reissner-Nordström solutions) are 
simply expressed in these coordinates. This will facilitate a discussion of approximate solutions as well as lead to some general properties. In Sec. 2 we will discuss properties common to all black hole solutions, while in Sec. 3 we will consider separately black holes of different sizes. In Sec. 4 we will present our numerical results. For this purpose, it is more convenient to choose the following form of the metric

$$
d s^{2}=-f d t^{2}+\frac{d r^{2}}{h}+r^{2} d \Omega
$$

where $f$ and $h$ are functions of $r$ only. With this form of the metric, $f$ can be solved for explicitly, and the $h$ equation becomes first order instead of second order. Sec. 5 contains some concluding remarks.

\section{General Properties}

Choosing the form of the metric (1.3), the dilaton equation $(\sqrt{1.2 b})$ and three nontrivial components of the metric equation $(1.2 d)$ become

$$
\begin{aligned}
\left(R^{2} \lambda \phi^{\prime}\right)^{\prime} & =m^{2} R^{2} \phi-\frac{Q^{2} e^{-2 \phi}}{R^{2}} \\
\left(R^{2} \lambda^{\prime}\right)^{\prime} & =-2 m^{2} R^{2} \phi^{2}+\frac{2 Q^{2} e^{-2 \phi}}{R^{2}}, \\
\left(\lambda\left(R^{2}\right)^{\prime}\right)^{\prime} & =2-2 m^{2} R^{2} \phi^{2}-\frac{2 Q^{2} e^{-2 \phi}}{R^{2}}, \\
0 & =R^{\prime \prime}+R\left(\phi^{\prime}\right)^{2}
\end{aligned}
$$

These four equations are not all independent. Eqs. (2.1d) and (2.1d) can be combined to yield a single equation with no second derivatives.

When $m=0$ these equations have a simple exact black hole solution [3] [4]

$$
\lambda=\left(1-\frac{2 M}{r}\right), \quad R^{2}=r^{2}\left(1-\frac{Q^{2} e^{-2 \phi_{\infty}}}{M r}\right), \quad e^{-2 \phi}=e^{-2 \phi_{\infty}}\left(1-\frac{Q^{2} e^{-2 \phi_{\infty}}}{M r}\right)
$$

where $M$ is the mass and $\phi_{\infty}$ is the value of the dilaton at infinity. The metric is identical to Schwarzschild except that the area of the spheres of spherical symmetry are reduced by

an amount depending on the charge. This area goes to zero at $r=\frac{Q^{2} e^{-2 \phi_{\infty}}}{M}$ which is the curvature singularity. In the extremal limit, $Q^{2}=2 M^{2} e^{2 \phi_{\infty}}$, the horizon itself becomes 
singular. However, when we conformally rescale to the string metric, one finds that the extremal limit is given by

$$
\widehat{d s}^{2}=-d t^{2}+\left(1-\frac{\sqrt{2}|Q|}{r}\right)^{-2} d r^{2}+r^{2} d \Omega
$$

where we have absorbed the constant $e^{\phi_{\infty}}$ into a redefinition of $r$ and $t$. This metric has neither a horizon nor a curvature singularity. The surfaces of constant time are asymptotically flat, and approach an infinite cylinder as $r$ approaches $\sqrt{2}|Q|$.

We begin our discussion of black holes with $m \neq 0$ by deriving a few general properties of these solutions. As boundary conditions at infinity, we require that the metric be asymptotically flat and the dilaton vanish. (Since we have chosen the minimum of the dilaton potential to be at $\phi=0$, one cannot shift the dilaton at infinity without destroying asymptotic flatness, unlike the massless case.) In particular, at infinity, $R$ must be equal to $r$. So integrating (2.1d) from any $r$ to infinity, we get $R^{\prime}(r)=1+\int_{r}^{\infty}\left(\phi^{\prime}\right)^{2} R d \tilde{r}$. In particular, $R^{\prime} \geq 1$ everywhere and $R$ is monotonically increasing. This shows that there are no wormholes outside the horizon in the Einstein metric.

Now consider the behavior of the dilaton outside the horizon. We first show that the dilaton must be positive on the horizon. Multiplying the dilaton equation by $\phi$ yields

$$
\left(\phi R^{2} \lambda \phi^{\prime}\right)^{\prime}=R^{2} \lambda \phi^{\prime 2}+m^{2} R^{2} \phi^{2}-\phi Q^{2} e^{-2 \phi} / R^{2}
$$

If $\phi$ were negative on the horizon one could integrate (2.4) from the horizon out to $\phi=$ 0. The left hand side vanishes and the right hand side is positive definite, yielding a contradiction. Next, it follows immediately from the dilaton equation that at a negative extremum, $R^{2} \lambda \phi^{\prime \prime}<0$. This means that if the dilaton ever becomes negative, it must continue to decrease. It cannot have a local minimum with $\phi<0$. Since the dilaton is positive at the horizon and zero at infinity, it must remain nonnegative. Finally, one can show that the dilaton is monotonically decreasing outside the horizon.2 Suppose $\phi$ has a local maximum. Then the left hand side of eq. (2.1a) must be negative. This implies that

$$
m^{2} \phi e^{2 \phi}<Q^{2} / R^{4}
$$

Since $\phi$ is decreasing as one moves in, the left hand side is decreasing while the right hand side is increasing. So the inequality remains valid everywhere between the local

2 We thank Ruth Gregory for suggesting this argument. 
maximum and the horizon. ( $\phi$ cannot have a local minimum since that would require that the inequality be reversed.) This leads to a contradiction at the horizon, since the left hand side of (2.1a) is $R^{2} \lambda^{\prime} \phi^{\prime}$ at the horizon, which is positive if $\phi^{\prime}>0$. This violates the inequality (2.5). Since $\phi$ cannot have a local maximum, it must be monotonically decreasing outside the horizon.

Next, consider the solution in the region far from the black hole. Since the metric is asymptotically flat and $\phi$ vanishes at infinity, the dilaton equation (2.1a) reduces to

$$
\left(r^{2} \phi^{\prime}\right)^{\prime}-m^{2} r^{2} \phi=-Q^{2} / r^{2}
$$

For large $r$ the derivative term is negligible and the solution is

$$
\phi \approx \frac{Q^{2}}{m^{2} r^{4}}
$$

The massless dilaton falls off at infinity like $1 / r$, and a massive scalar field with localized sources falls off exponentially. Here we have both a mass and a source which is going to zero like a power of $1 / r$. These combine to give the somewhat unusual asymptotic behavior of the dilaton. Now consider the metric equations (2.1b) and (2.1c). The contributions to the right hand sides from the dilaton fall off like $1 / r^{6}$ which is much faster than the dilaton independent term $Q^{2} / r^{2}$. As a result, the leading order solution for $\lambda$ and $R$ is exactly the Reissner-Nordström solution. The first order corrections to Reissner-Nordström can be found by solving the equations perturbatively. One finds that

$$
\begin{aligned}
\lambda & \approx 1-\frac{2 M}{r}+\frac{Q^{2}}{r^{2}}-\frac{Q^{4}}{5 m^{2} r^{6}} \\
R & \approx r\left(1-\frac{2 Q^{4}}{7 m^{4} r^{8}}\right)
\end{aligned}
$$

Thus the corrections to the Reissner-Nordström solution are quite small for large $r$. We should caution the reader at this point that the full power series expansion of the solution around $r=\infty$ has zero radius of convergence, so one must be careful when including large numbers of higher order terms in (2.8). The approximations we have written down give the correct leading behavior.

Now we consider the behavior of the solutions near the singularity. Consider first the massless dilaton solution (2.2). Since the dilaton is diverging near the singularity at $r=\frac{Q^{2} e^{-2 \phi_{\infty}}}{M}$, it might appear that the effect of adding a nonzero mass will become important. However the dilaton only diverges logarithmically, and in (2.1) it appears 
multiplied by $R^{2}$ which vanishes linearly. (By contrast, the charge terms remain nonzero near the singularity.) The net effect is that the mass term becomes less important as one approaches the singularity. So the $m=0$ solution (2.2) becomes a better and better approximation to an $m \neq 0$ solution in this limit. However this is not sufficient to prove that the massive dilaton black holes approach the $m=0$ solution (2.2) near the singularity. One knows only that there are some solutions of the $m \neq 0$ equations which agree with (2.2) initially and start to deviate as one moves out. There is no guarantee that the resulting solutions will be asymptotically flat, or even have an event horizon.

We will see in Sec. 4 that the massive dilaton black holes do approach a solution of the $m=0$ equations near the singularity, but not necessarily the one given in eq. (2.2). The solution (2.2) was determined by the requirement that it have a regular event horizon and be asymptotically flat. Near the singularity, these conditions do not apply. The general solution to equations (2.1) with $m=0$ can be found in closed form. This solution depends on five free parameters, but it is convenient to express it in terms of six parameters subject to one condition. Let $x=\left(\frac{r-r_{+}}{r-r_{-}}\right)^{\frac{1}{2}}$. Then the solution is given by

$$
\lambda=\frac{x^{\sqrt{2-c_{1}^{2}}}}{c_{2} x^{c_{1}}+c_{3} x^{-c_{1}}}, \quad R^{2}=\left(r-r_{+}\right)\left(r-r_{-}\right) / \lambda, \quad e^{2\left(\phi-\phi_{\infty}\right)}=x^{2 \sqrt{2-c_{1}^{2}}} / \lambda
$$

where $c_{1}^{2} c_{2} c_{3}\left(r_{+}-r_{-}\right)^{2}+2 Q^{2} e^{-2 \phi_{\infty}}=0$. The five free parameters are just what one expects for the general solution to three second order differential equations and one constraint. However two of these parameters are essentially pure gauge. The form of the metric (1.3) is invariant under shifting $r$ by a constant, and rescaling $r \rightarrow c r, \lambda \rightarrow c^{2} \lambda$ and $t \rightarrow t / c$. So there is really a three dimensional space of physically different solutions. When $c_{1}=1$ the solution (2.9) reduces to the standard massless dilaton black hole, with the two remaining parameters corresponding to $M$ and $\phi_{\infty}$. When $c_{1} \neq 1$ the solution (2.9) does not have a regular event horizon. One can verify that unless $c_{1}$ is close to $\sqrt{2}$, the effect of a mass term becomes negligible near the singularity for any of these solutions.

\section{Approximate Solutions}

There are three distinct types of black hole solutions depending on the size of the black hole compared to the Compton wavelength of the dilaton. 


\subsection{Large Black Holes}

First consider a black hole with $M m \gg 1$ i.e. a black hole with Schwarzschild radius much larger than the Compton wavelength of the dilaton. We claim that the asymptotic solution (2.7), (2.8) found above remains close to the exact solution up to and past the event horizon. Outside the horizon, the maximum possible value of the correction terms to Reissner-Nordström is obtained when $Q^{2} \approx M^{2}$ and $r \approx M$. Even in this case, the correction to $\lambda$ is of order $(M m)^{-2}$ and the correction to $R$ is of order $(M m)^{-4}$. Since these correction terms were derived under the assumption that $\phi \approx Q^{2} / m^{2} r^{4}$ we must also verify that this remains valid everywhere outside the horizon. This solution for $\phi$ followed from the fact that $\phi \ll 1$ and the derivative term in (2.6) was negligible. The first condition is clearly satisfied until one is well inside the black hole. The second condition will remain true until

$$
\left[\left(r^{2}-2 M r+Q^{2}\right) \phi^{\prime}\right]^{\prime} \approx Q^{2} / r^{2}
$$

Using the approximate value of $\phi$ from (2.7) one finds that the approximation is valid as long as $m r>(m M)^{1 / 3}$ or $m r>(m Q)^{1 / 2}$ whichever is larger. Since the event horizon occurs when $r$ is of order $M$, this condition is also valid until well inside the black hole. (The second alternative is just the condition that $\phi$ be of order one.) For $Q^{2}$ of order $M^{2}$, the inner horizon of the Reissner-Nordström black hole is close to the event horizon. The above argument indicates that the solution will stay close to Reissner-Nordström even past the inner horizon. This suggests that large black holes with large charge coupled to a massive dilaton will have nonsingular inner horizons even though massless dilaton black holes do not. 3 The numerical solutions in Section 4 will confirm this. If the charge is much less than the mass, the approximation breaks down before the Reissner-Nordström inner horizon is reached. In this case, we will find numerically that the solutions have only one horizon.

For a Reissner-Nordström black hole, the extremal limit occurs when the inner and outer horizons coincide. This happens when $Q^{2}=M^{2}$ and the horizon is $r_{0}=M$. Since the large black hole is quite similar to Reissner-Nordström until well within the inner horizon for $Q^{2} \sim M^{2}$, the extremal limit of the large black hole is qualitatively similar to the Reissner-Nordström extremal limit. The presence of a massive dilaton does however

3 Rotating black holes with a massless dilaton do have an inner horizon [8]. 
shift the extremal limit away from $Q^{2}=M^{2}$. With the parameterization in (1.3), a horizon occurs whenever $\lambda=0$, so using (2.8), the condition for a horizon is

$$
r^{2}-2 M r+Q^{2}=\frac{Q^{4}}{5 m^{2} r^{4}}
$$

We can calculate the deviations from the Reissner-Nordström limit by fixing $M$ and perturbing $Q^{2} \rightarrow M^{2}+\delta Q^{2}$ and $r_{0} \rightarrow M+\delta r$. To leading order in $1 /(m M)$, (3.2) gives $\delta Q^{2}=1 / 5 m^{2}$, or

$$
\frac{Q_{\mathrm{ext}}^{2}}{M^{2}}=1+\frac{1}{5 M^{2} m^{2}}
$$

Thus in the extremal limit, the charge is always larger than the mass. The maximum charge for given mass increases as the black hole becomes smaller. If the charge is increased beyond the extremal limit, the horizons disappear and a naked singularity occurs.

The electromagnetic and gravitational forces between two extremal Reissner-Nordström black holes (of the same sign) exactly cancel. We have seen that large extremal string black holes approach Reissner-Nordström asymptotically and always have $Q_{\text {ext }}^{2}>M^{2}$. This increases the magnetic repulsion, so two extremal string black holes at large separation will repel each other. This fact has the following important consequence. It is energetically favorable for one large extremal black hole to split into several smaller ones. This follows from (3.3) since the mass of a single extremal black hole with charge $Q$ is $M_{1}=\left(Q^{2}-1 / 5 m^{2}\right)^{\frac{1}{2}}$ while the mass for $n$ widely separated extremal black holes with charge $Q / n$ is

$$
M_{n}=n\left[\left(\frac{Q}{n}\right)^{2}-\frac{1}{5 m^{2}}\right]^{\frac{1}{2}}=\left[Q^{2}-\frac{n^{2}}{5 m^{2}}\right]^{\frac{1}{2}} .
$$

Clearly, $M_{n}$ is a decreasing function of $n$. (We cannot decrease $M_{n}$ to zero since this formula is only valid when $m M_{n} \gg 1$.) Of course this breakup cannot occur classically since horizons cannot bifurcate [9]. But it will occur through quantum tunneling, if there is no infinite barrier to prevent it. One can argue against the presence of an infinite barrier by finding an instanton which describes this tunneling event. This is currently under investigation.

The possibility that extremal Reissner-Nordström or massless dilaton black holes can quantum mechanically bifurcate has recently been discussed [10] [11]. Since there is no force between these black holes, the analogous calculation would show $M_{n}$ is independent of $n$. The different states are degenerate in energy. Whether or not tunneling is possible 
in this case, we have shown that it is much more likely to occur when there is a massive dilaton.

Although we have considered extremal black holes, it should be clear that the same conclusion applies to nearly extremal black holes. The fact that $M_{n}<M_{1}$ will not be altered if the charge on each black hole is decreased slightly. The main difference between extremal and nonextremal black holes, is that the nonextremal holes will quantum mechanically emit ordinary Hawking radiation. Since the extremal black holes have a degenerate horizon, their Hawking temperature vanishes. As we have just seen, this is not sufficient to conclude that they are quantum mechanically stable. This strongly suggests that the extremal black holes are not supersymmetric.

\subsection{Small Black Holes}

Now consider the case $m M \ll 1$ when the black hole is small compared to the Compton wavelength of the dilaton. In the asymptotically flat region far from the hole, the dilaton satisfies eq. (2.6) and has the asymptotic solution (2.7). Since $m M \ll 1$, there is now a region $(m M)^{1 / 3} \ll m r \ll 1$ where the metric is still essentially flat, but the dilaton is no longer given by (2.7). (The lower limit comes from requiring that the terms in the dilaton equation depending on $M$ be negligible.) The exact solution to (2.6) can be found in terms of the exponential integral function, but for our purposes it suffices to notice that for $m r \ll 1$, the solution is approximately

$$
\phi \approx-\frac{Q^{2}}{2 r^{2}}+\frac{A}{r}
$$

where $A$ is an arbitrary constant. We showed earlier that the dilaton cannot be negative outside the event horizon. Thus, in order to have a solution describing a black hole, $A$ must be sufficiently large that the second term dominates the first. But this is precisely the behavior of a massless dilaton at large distances from a black hole. One expects that the solution will now resemble the massless dilaton black hole, at least until the dilaton becomes large.

When considering how the massless dilaton black hole matches onto the ReissnerNordström solution, it is perhaps worth mentioning that the two solutions are actually more similar than they may appear. As we have mentioned, there is a residual gauge freedom in the form of the metric (1.3) corresponding to shifting $r$ by a constant. If we 
introduce a new radial coordinate $r=\tilde{r}+Q^{2} / 2 M$, then the massless dilaton solution (2.2) (with $\phi_{\infty}=0$ ) becomes

$$
\lambda \approx 1-\frac{2 M}{\tilde{r}}+\frac{Q^{2}}{\tilde{r}^{2}}+O\left(\tilde{r}^{-3}\right) \quad R^{2} \approx \tilde{r}^{2}\left[1-\left(\frac{Q^{2}}{2 M \tilde{r}}\right)^{2}\right]+O\left(\tilde{r}^{-1}\right)
$$

which shows that the solutions really differ only at order $1 / r^{2}$.

In the general massless dilaton solution (2.9), a horizon exists only when $c_{1}=1$. Since we expect that small black holes resemble the massless dilaton solution, $c_{1}$ must be 1 up to small corrections. Small deviations from $c_{1}=1$ are allowed since the corrections from the dilaton mass can compensate and still permit a horizon to form. We will see in the next section that for nonextremal black holes, these small deviations from $c_{1}=1$ do not vanish as one approaches the singularity. Thus, even though the mass terms become negligible near the singularity, the solution is not approaching (2.2). However, in the extremal limit, the horizon itself approaches the singularity. So the small corrections to $c_{1}$ must be getting smaller and smaller. Thus, up to the coordinate freedom of shifting $r$ and rescaling, the solution approaches the standard massless solution (2.2). In the string metric, the extremal small black holes will disappear down an infinite throat. One might be tempted to conclude that the extremal value of the charge for any small black hole is given by the massless dilaton result $Q_{\text {ext }}^{2}=2 M^{2}$ (using the fact that the dilaton must vanish at infinity). However, the parameters $M$ and $\phi_{\infty}$ in (2.2) do not in general correspond exactly to the asymptotic values of the mass or dilaton. This should be the case only in the limit of very small black holes. Thus we expect that the extremal value of the charge for small black holes has the form

$$
\frac{Q_{\mathrm{ext}}^{2}}{M^{2}}=2-\alpha m^{2} M^{2}+\mathcal{O}\left(m^{4} M^{4}\right)
$$

where $\alpha$ is an undetermined positive constant. It is simple to check that extremal black holes with charge given by (3.7) are unstable in exactly the same manner as the large black holes.

\subsection{Black Holes with $M m \approx 1$}

Some analytic results about black holes with $M m \sim 1$ can be obtained as follows. We have seen that the extremal limit of a large black hole has a degenerate horizon, while the extremal limit of small black holes has a singular horizon (which in the string metric 
corresponds to an infinite throat). Let us consider the condition for the existence of a degenerate horizon. If both $\lambda$ and $\lambda^{\prime}$ vanish at a point $r_{d}$, then from $(2.1 d)$ and $(2.1 d)$,

$$
\begin{aligned}
& m^{2} R_{d}^{2} \phi_{d}=\frac{Q^{2} e^{-2 \phi_{d}}}{R_{d}^{2}} \\
& m^{2} R_{d}^{2} \phi_{d}^{2}+\frac{Q^{2} e^{-2 \phi_{d}}}{R_{d}^{2}}=1
\end{aligned}
$$

where $R_{d}$ and $\phi_{d}$ are the values of $R$ and $\phi$ at $r_{d}$. Combining these equations yields

$$
\begin{gathered}
R_{d}^{2}=\frac{1}{m^{2} \phi_{d}\left(1+\phi_{d}\right)} \\
\frac{e^{2 \phi_{d}}}{\phi_{d}\left(1+\phi_{d}\right)^{2}}=m^{2} Q^{2}
\end{gathered}
$$

Eq. (3.10) has two different regimes depending on the value of $m^{2} Q^{2}$. The left hand side reaches its minimum value of $e^{2} / 4$ when $\phi_{d}=1$. Thus degenerate horizons cannot occur when $m^{2} Q^{2}<e^{2} / 4$. In other words, black holes with $m^{2} Q^{2}<e^{2} / 4$ cannot have an extremal limit similar to the Reissner-Nordström solution. Instead, they will behave like the massless dilaton solution. When $m^{2} Q^{2}$ is larger than the minimum value, there are two solutions for $\phi_{d}$. They can be distinguished by looking at $\lambda_{d}^{\prime \prime}$ which from (2.10) is

$$
R_{d}^{2} \lambda_{d}^{\prime \prime}=2 m^{2} R_{d}^{2} \phi_{d}\left(1-\phi_{d}\right)
$$

Since $\lambda>0$ outside the degenerate horizon (assuming it is the event horizon), we require $\lambda_{d}^{\prime \prime}>0$ which implies $\phi_{d}<1$. For a large black hole with $m^{2} Q^{2} \gg 1$, the solution of (3.10) for $\phi_{d}$ is approximately $\phi_{d}=(m Q)^{-2}$. This agrees with our earlier solution $\phi=Q^{2} / m^{2} r^{4}$ when we use the fact that $r=Q$ at the horizon of degenerate large black holes.

The critical value $m^{2} Q^{2}=e^{2} / 4$ is quite interesting. We see from (3.11) that $\lambda_{d}^{\prime \prime}=0$ when $\phi_{d}=1$, so that the degenerate horizon is actually triply degenerate at the critical value (the higher derivatives are nonzero). This may indicate that three horizons are coming together at this point instead of just the two that come together when $m^{2} Q^{2}>$ $e^{2} / 4$. The possibility of black holes with three horizons has been discussed in the literature [12]. However, we see no evidence of this in the numerical simulations described in the next section. Because of the singularities in the equations, the numerical simulations behave very poorly right near this critical value, so we cannot rule out spacetimes with three horizons. However, it is clear that this is not the generic case for $m^{2} Q^{2}>e^{2} / 4$. 
All of the preceding discussion has been in term of the Einstein metric. Strings couple instead to the string metric, which is equal to the Einstein metric multiplied by $e^{2 \phi}$. So the radius of the spheres of spherical symmetry is $R e^{\phi}$. We have shown that $R$ is monotonically increasing and $\phi$ is monotonically decreasing outside the horizon. So $\left(R e^{\phi}\right)^{\prime}$ need not have a definite sign. For the massless dilaton black holes one finds that $R e^{\phi}$ is an increasing function of $r$, which means that the spacetime is similar to the Schwarzschild solution. The maximally extended spacetime has a wormhole at the horizon, but an observer falling in sees the spheres monotonically decrease in area. When $m \neq 0$, the situation is different: $R e^{\phi}$ can decrease outside the horizon of certain black holes. Since $R e^{\phi}$ is certainly increasing near infinity, the spacetime describes a wormhole outside the event horizon.

To see this, we will need a relation between $R^{\prime}$ and $\phi^{\prime}$ valid for any degenerate horizon. Taking the derivative of the dilaton equation of motion and evaluating at a degenerate horizon yields

$$
R_{d}^{\prime}=-R_{d} \phi_{d}^{\prime} \frac{1+2 \phi_{d}^{2}}{4 \phi_{d}} .
$$

Since $R^{\prime}>1$ everywhere, we see that $\phi$ must decrease away from a degenerate horizon as well as a regular horizon. Using (3.12) we obtain:

$$
\left(R e^{\phi}\right)_{d}^{\prime}=e^{\phi_{d}}\left(R^{\prime}+R \phi^{\prime}\right)_{d}=R_{d} \phi_{d}^{\prime} e^{\phi_{d}}\left[1-\frac{1+2 \phi_{d}^{2}}{4 \phi_{d}}\right] .
$$

Since $\phi_{d}^{\prime}<0$, if $\phi_{d}$ is either very large or very small the quantity in brackets is negative and $\left(R e^{\phi}\right)_{d}^{\prime}>0$ as expected. However for $1-1 / \sqrt{2}<\phi_{d}<1+1 / \sqrt{2}$ one has $\left(R e^{\phi}\right)_{d}^{\prime}<0$. In this case the string metric describes a wormhole outside the event horizon. A string falling in will pass through the throat of the wormhole before entering the black hole.

The above local argument is not complete in that we have not yet shown that one can evolve the spacetime outward from the wormhole and obtain an asymptotically flat region. This gap will be filled by the numerical solutions in the next section. Although we have found these wormholes by studying degenerate horizons, it should be clear that wormholes can exist outside regular event horizons as well. If one increases the mass slightly keeping the charge fixed, the throat of the wormhole will remain outside the horizon. 


\section{Numerical Results}

\subsection{General Method}

As mentioned earlier, for numerical results, it is convenient to use the following form of the Einstein metric

$$
d s^{2}=-f d t^{2}+h^{-1} d r^{2}+r^{2} d \Omega
$$

where $f$ and $h$ are functions of $r$ only. This form of the metric is justified since $R$ is monotonically increasing. As we have just seen, it would not be wise to assume this form for the string metric. The coordinates would then break down outside the horizon at the throat of the wormhole. The $t t$ and $r r$ components of Einstein's equation now become

$$
\begin{aligned}
& -r h^{\prime}+1-h=r^{2} h{\phi^{\prime}}^{2}+r^{2} m^{2} \phi^{2}+\frac{Q^{2} e^{-2 \phi}}{r^{2}}, \\
& \frac{r h f^{\prime}}{f}-1+h=r^{2} h{\phi^{\prime}}^{2}-r^{2} m^{2} \phi^{2}-\frac{Q^{2} e^{-2 \phi}}{r^{2}} .
\end{aligned}
$$

These are both first order equations. The first is the standard constraint equation and hence is independent of $f$. The remaining component of Einstein's equation will be satisfied if the stress tensor is conserved, which will hold if the dilaton satisfies its field equation:

$$
h^{1 / 2}\left[h^{1 / 2} r^{2} \phi^{\prime}\right]^{\prime}+\frac{r^{2} h \phi^{\prime} f^{\prime}}{2 f}=r^{2} m^{2} \phi-\frac{Q^{2} e^{-2 \phi}}{r^{2}} .
$$

One can solve for $f$ by adding eqs. (4.2a) and (4.2b). The result is

$$
f=h e^{2 \int r \phi^{\prime 2}} .
$$

Thus $f$ vanishes if and only if $h$ does. We can now eliminate $f$ and $h^{\prime}$ from the dilaton equation (4.3) to obtain:

$$
h r \phi^{\prime \prime}+\phi^{\prime}\left[1+h-r^{2} m^{2} \phi^{2}-\frac{Q^{2} e^{-2 \phi}}{r^{2}}\right]-r m^{2} \phi+\frac{Q^{2} e^{-2 \phi}}{r^{3}}=0 .
$$

The problem of finding black hole solutions thus reduces to solving (4.2a) and (4.5). One can eliminate $m$ from these equations by using the fact that they are invariant under the following rescaling: $r \rightarrow c r, m \rightarrow m / c, Q \rightarrow c Q$. We therefore set $m=1$. This corresponds to measuring distances in units of the Compton wavelength of the dilaton. Since the right hand side of $(4.2 a)$ is positive definite, it follows that $h$ cannot have an extremum with $h>1$. If $h$ ever increases larger than one, it must continue to increase. 
We now briefly consider the asymptotic form of the solutions near the singularity. Translating the massless dilaton solution (2.2) near the singularity into our new coordinates yields

$$
h=a / r^{2} \quad, \quad e^{-2 \phi}=b r^{2}
$$

where $a$ and $b$ are constants related to $M$ and $Q$. More generally, let us try a solution of the form

$$
h=c / r^{\alpha} \quad, \quad e^{-2 \phi}=d r^{2 \beta} .
$$

where $c, d, \alpha$ and $\beta$ are constants. The mass terms and charge terms in (4.2a) and (4.5) will both be negligible near the singularity if $\alpha$ is positive and larger than $2(1-\beta)$. In this case, the leading order terms in (4.2a) diverge like $r^{-\alpha}$ and vanish if $\alpha=1+\beta^{2}$. Combining this with the condition $\alpha>2(1-\beta)$ yields $\beta>\sqrt{2}-1$. The leading order terms in (4.5) diverge like $r^{-\alpha-1}$ and are automatically satisfied. These solutions can be characterized by the constant $\beta=-r \phi^{\prime}$. By transforming the general massless solution (2.9) near the singularity into our new coordinates, one can relate $\beta$ to the constant $c_{1}$. The familiar massless dilaton black hole has $\beta=1$. We will see that the massive dilaton black holes typically approach this more general solution near the singularity with $\beta \neq 1$. Since the radius of the spheres in the string metric is $r e^{\phi} \sim r^{1-\beta}$, when $\beta>1$, the spheres become infinitely large near the singularity.

We now need to numerically integrate the equations (4.2a) and (4.5). Since eq. (4.2a) is first order in $h$ and eq. (4.5) is second order in $\phi$, we must impose boundary conditions at a point $r_{0}$ by specifying $h\left(r_{0}\right), \phi\left(r_{0}\right)$ and $\phi^{\prime}\left(r_{0}\right)$. It might seem straightforward to use the known asymptotic behavior of the solutions to fix these three parameters at some large $r$ and then integrate in. However this fails for the following reason. At large $r$, the dilaton equation has both exponentially growing and exponentially decaying solutions. Any small numerical error in the initial values of $h\left(r_{0}\right), \phi\left(r_{0}\right)$ and $\phi^{\prime}\left(r_{0}\right)$ will correspond to a small error in the coefficient of the mode which grows exponentially as one evolves inward. The result is that numerical integration is extremely unstable and one cannot reliably integrate in to the horizon. To avoid this, we use a technique which actually turns the above instability to our advantage.

The first step is to reduce the number of free parameters. A horizon, where $h\left(r_{0}\right)=0$, is a singular point of the equations (4.2a) and (4.5). Since $\phi^{\prime \prime}\left(r_{0}\right)$ appears multiplied by

4 We thank P. Bizon for bringing this technique to our attention. It was used e.g. in [13]. 
$h\left(r_{0}\right)$ in eq. (4.5), at a horizon, eq. (4.5) becomes a constraint relating $\phi^{\prime}\left(r_{0}\right)$ with $\phi\left(r_{0}\right)$. This reduces the number of parameters needed to specify the solution by one. Since the horizon is a singular point of the equations however, we cannot begin numerical integration there. Instead, we must do a power series expansion of the solution around the horizon, and start the numerical integration slightly outside. In other words, choose a point $r_{0}$. Expand the dilation and metric around the horizon using

$$
\begin{aligned}
& h(r)=\left(r-r_{0}\right) \sum_{i=0}^{\infty} h_{i}\left(r-r_{0}\right)^{i}, \\
& \phi(r)=\sum_{i=0}^{\infty} \phi_{i}\left(r-r_{0}\right)^{i} .
\end{aligned}
$$

Using this expansion in eq. (4.2a) and (4.5), one sees that the solution has one free parameter, $\phi\left(r_{0}\right)=\phi_{0}$. As happens with most power series in this problem, the expansion (4.8) seems to be only an asymptotic expansion, so in the numerical integrations, we will keep only the first three terms. Even the low order terms are quite lengthy, so we will not present them explicitly here.

The numerical integration now proceeds as follows. Fix $Q$, and select a value for $r_{0}$. This fixes the area of the horizon which is equivalent to fixing a mass $M$. (Recall that we have set $m=1$.) Choose a small parameter $\epsilon$ which determines the starting point of the integration $r_{0}+\epsilon$. Now choose a value of $\phi_{0}$ (this determines $h\left(r_{0}+\epsilon\right), \phi\left(r_{0}+\epsilon\right)$, and $\left.\phi^{\prime}\left(r_{0}+\epsilon\right)\right)$ and integrate outwards. As we have seen, the equations (4.2a) and (4.5) are extremely numerically unstable, which makes the outwards integration tricky. One can use this instability to determine the correct value for $\phi_{0}$. The homogeneous solution to the flat space dilaton equation (2.6) has an exponentially growing solution. If $\phi_{0}$ is chosen to be too small, then the undesired exponential mode mixes into the real solution with a negative coefficient. As $r$ increases, this drives $\phi(r)$ off towards $-\infty$. As discussed in Sec. 2, $\phi(r)$ cannot be negative outside an event horizon. Thus it is easy to tell if the initial value of $\phi_{0}$ was too small. Conversely, if $\phi_{0}$ is initially chosen to be too large, then the undesired exponential mode enters with a positive coefficient, which drives $\phi(r)$ towards $+\infty$. As we saw in Sec. $2, \phi(r)$ must monotonically decrease outside the horizon, so it is also easy to tell if one has chosen $\phi_{0}$ too large. Once one has found a $\phi_{0}$ that is too large and one that is too small, it is straightforward to test their mean value to see whether it is too large or too small. One can then iterate the procedure until a value of $\phi_{0}$ is found that approximates the desired asymptotic behavior. Because of numerical errors, 
it is impossible to completely eliminate the instability, but it is possible to determine the correct value of $\phi_{0}$ to extremely high precision.

To obtain the interior solution, one uses the value of $\phi_{0}$ just obtained and integrates inward starting at $r_{0}-\epsilon$. The crucial point is that $\phi_{0}$ can be determined to sufficient accuracy so that the interior solution is insensitive to the residual error. For small black holes, one can integrate the equations to arbitrarily close to the singularity. For black holes with an inner horizon, the equations become singular at the inner horizon, and the integration breaks down there. However, one can integrate to quite close to the inner horizon, and determine the value of $\phi$ at the inner horizon. This can be used as a new $\phi_{0}$ to integrate inwards to the singularity. One must be careful with this procedure, because the value of $\phi$ at the inner horizon cannot be determined as precisely as the value of $\phi$ on the event horizon, and the solutions near the singularity are sensitive to the value of $\phi_{0}$ used at the inner horizon.

To perform the numerical integration, we used the extremely powerful differential equation solver lsoda.f [14]. Using this Fortran program, it takes very little time to determine the correct value of $\phi_{0}$ to up to fourteen decimal places in all but the most pathological situations.

\subsection{Specific Numerical Examples}

Fig. 1: Integrating the dilaton outside an event horizon with $r_{0}=100$ and $Q=60$. The upper line is $\phi_{0}=3.5988199312 \times 10^{-5}$, the middle (dashed) line is $Q^{2} / r^{4}$, and the bottom line is $\phi_{0}=3.5988199311 \times 10^{-5}$. 
For our first example, we take $r_{0}=100$ and $Q=60$. This corresponds to a mass $M=68$, which can be determined from the fact that outside the horizon, this solution is very close to the Reissner-Nordström solution. These parameters should correspond to a large black hole with two horizons. In fig. 1, we show what happens when one integrates outwards. Two integrations are shown, with slightly different values of $\phi_{0}$. One is diverging upwards, and the other is diverging downwards. The dashed line in the middle is just the approximation from eq. (2.7), showing that the dilaton is quite close to the expected value outside the event horizon (the leading order terms in (2.7) and (2.8a) are still correct in this coordinate system). The correct value for $\phi_{0}$ lies between the two given values. Notice that the outwards integration does not go far before the instability sets in. This is not a serious problem, since we have a good approximation to the solution outside the horizon, (2.7) and (2.8). The important point is that one can integrate far enough to determine $\phi_{0}$ to high precision. Integrating inwards from the event horizon works well, and the two values of $\phi_{0}$ give essentially indistinguishable answers inside the black hole, at least up to the inner horizon.

Fig. 2: $h(r)$ between the horizons of a black hole with $r_{0}=100$ and $Q=60$.

The metric function $h(r)$ for this black hole inside the event horizon is shown in fig. 2 . It agrees almost precisely with the approximate solution in $(2.8 a)$. The deviations are too small to show up on the plot. The inner horizon appears at $r=35.976$. This confirms that black holes with massive dilatons can have inner horizons. The dilaton is shown in fig. 3. It also has essentially no deviation from the approximate value (2.7). 
Fig. 3: $\phi(r)$ between the horizons of a black hole with $r_{0}=100$ and $Q=60$.

Fig. 4: $\phi(r)$ inside the inner horizon. The upper line is the numerical result with $\phi_{0}=2.1517496625 \times 10^{-3}$, the lower line is $\phi_{0}=2.1517496620 \times 10^{-3}$, and the dashed line is the expected approximate value from (2.7).

Continuing the integration inside the inner horizon is more difficult. Because the equations become singular at the inner horizon, it is simple to determine its position. Unfortunately, numerical errors make the precise value of $\phi$ difficult to determine. A good approximation can be found, but this seems to be insufficient to integrate up to the singularity, as the interior solution exhibits an instability similar to the one outside the event horizon. (This instability does not occur when the inner horizon is absent, and seems to be related to $h>0$.) An example of this is shown in fig. 4, where two similar values of $\phi$ on the inner horizon are used to begin integration. In most cases, one can push the instability to inside the point where $h(r)>1$. We have seen that once $h(r)>1$, it must increase to $+\infty$, so there can be no third horizon. 
Fig. 5: $h(r)$ for a black hole with $r_{0}=100$ and $Q=20$, which corresponds to $M=52$. The solid line is the numerical result, and the dashed line is the approximation from (2.8a).

Our next example is a large black hole which has no inner horizon. The event horizon is again chosen to be at $r_{0}=100$, but the charge is now $Q=20$. These parameters correspond to a black hole with mass $M=52$. Again, it is not possible to integrate outwards very far from the event horizon, but $\phi_{0}$ can be determined to high accuracy. We find that with these parameters, the dilaton at the horizon is $\phi_{0}=3.998433496484 \times 10^{-6}$, which is excellent agreement with the approximate answer given by $Q^{2} / r^{4}=4 \times 10^{-6}$. Unlike the previous example, the solution inside the event horizon is quite stable under small changes in $\phi_{0}$ all the way to the singularity. In fig. 5, we compare the metric function $h(r)$ inside the horizon with the approximate solution given in equation (2.8a). The agreement is excellent in to $r \approx 5$. The small increase in $h(r)$ around $r=4$ is where the Reissner-Nordström solution has a inner horizon.

In fig. 6, we show the dilaton inside the event horizon of the same black hole. Since the dilaton varies across a wide range, we have actually plotted $\log \phi$. For comparison, we have also plotted $Q^{2} / r^{4}$. The dilaton agrees with the approximate solution until well inside the event horizon, but as expected, starts diverging inside $r \approx 10$ (this deviation is not numerical error, but due to the breakdown of the approximation (2.7)).

The behavior of this black hole near the singularity is quite illustrative. The behavior of $r \phi^{\prime}(r)$ is shown in fig. 7. As discussed earlier, if the solution were approaching the massless solution (2.2), near the singularity, $r \phi^{\prime} \rightarrow-1$. We can see clearly that this is not the case. Instead, $r \phi^{\prime} \rightarrow-2.79$. This corresponds to a solution of the type (4.7), so the mass terms are becoming negligible near the singularity. (As expected, $h$ diverges 
Fig. 6: $\log \phi(r)$ for a black hole with $r_{0}=100$ and $Q=20$. The solid line is the numerical solution, and the dashed line is the approximate solution from (2.7).

Fig. 7: The behavior of $r \phi^{\prime}(r)$ near the singularity when $r_{0}=100$ and $Q=20$.

like $r^{-\left(1+\beta^{2}\right)}$ with $\beta=2.79$.) However, the solution is not approaching the massless solution (2.2). Instead, it is approaching a more general solution to the massless equations. The metric function $h(r)$ for a small black hole is shown in fig. 8. This black hole has $r_{0}=.01$ and $Q=.001$. For small black holes, one can integrate outwards considerably farther before the numerical instability sets in. The mass $M$ of the black hole can be easily determined to be $M=.00504976$ by integrating out to a large enough $r$ so that the asymptotic approximation (2.8a) is valid.

In fig. 9, we show the dilaton outside the event horizon for the same small black hole, along with the asymptotic solution (2.7) and the massless solution (2.2) for this $Q$ and $M$ (setting $\phi_{\infty}=0$ to match properly at the horizon, and transforming into the new 
Fig. 8: $h(r)$ outside the event horizon of a small black hole with $r_{0}=.01$ and $Q=.001$.

Fig. 9: $\log \phi(r)$ outside the event horizon of a black hole with $r_{0}=.01$ and $Q=.001$. The solid line is the numerical result, the upper dashed line is the massless solution, and the lower dashed line is the asymptotic solution.

coordinates (4.1)). As expected, the dilaton is falling off much faster than in the massless dilaton solution. At large $r$ it clearly approaches the asymptotic solution.

Inside the event horizon of this small black hole, the situation is different. Now the numerical solution behaves almost precisely like the solution with a massless dilaton. The dilaton inside is shown in fig. 10. The dilaton for the $m=0$ solution is indistinguishable in this plot. This confirms that small black holes behave similarly to the $m=0$ solutions. In this case, one finds $r \phi^{\prime} \rightarrow-1.00000097$ at the singularity, which is close to the $m=0$ value. The interior solution is stable under perturbations of $\phi_{0}$, so the slight deviation from $r \phi^{\prime} \rightarrow-1$ does seem to be real. Since this black hole is not extremal, some deviation 
Fig. 10: $\log \phi$ inside the event horizon of a small black hole with $r_{0}=.01$ and $Q=.001$.

might be expected. The fact that it is so small seems to be a reflection of the fact that $Q$ is quite small.

Fig. 11: $h(r)$ for a black hole with $r_{0}=.85$ and $Q=1.36455$.

Our next example is a medium sized black hole, with $r_{0}=.85$ and $Q=1.36455$. The metric function near the horizon is shown in fig. 11, and a more detailed picture is in fig. 12. From the asymptotic value of $h(r)$, we can determine the mass of this black hole to be $M=1.29283$. This black hole is close to the extremal limit, and also close to the triple point. Notice that $Q^{2} / M^{2}>1$, so this black hole is unstable to splitting into smaller, widely separated black holes. If we were to decrease $r_{0}$ or increase $Q$ by a small amount, the slight increase in $h(r)$ would touch $h=0$, causing an inner horizon. 
Fig. 12: An enlargement of $h(r)$ near the horizon for the medium black hole.

Fig. 13: The radius of spheres in the string metric, $r e^{\phi}$, is shown near the horizon for the medium black hole. The straight line is the position of the horizon.

The horizon for this black hole lies behind a wormhole in the string metric. In fig. 13, we show the radius of spheres in the string metric $r e^{\phi}$ as a function of $r$. Notice that $r e^{\phi}$ reaches a minimum outside of the event horizon, and increases inwards until turning over again farther in.

We demonstrate the approach to the extremal limit in fig. 14. This shows the results for a number of black holes, all with charge $Q=1$. The horizon for a black hole is picked to be at a particular $r_{0}$, and the value of $\beta=-\lim _{r \rightarrow 0} r \phi^{\prime}$ is then determined. Smaller values of $r_{0}$ correspond to black holes closer to the extremal limit. As can be seen in fig. 14, as the black hole approaches the extremal limit, $\beta \rightarrow 1$, which is the massless dilaton value. Thus, even medium black holes with a charge too small to have a degenerate horizon, will 
Fig. 14: $\beta$ versus $\log r_{0}$, the position of the event horizon, for black holes with charge $Q=1$.

have an infinite throat in the extremal limit. Notice that $\beta$ is becoming larger than one as one moves farther from the extremal limit. This is consistent with our earlier result that $\beta=2.79$ for a black hole with $Q=20$. As the extremal limit is approached, we can determine from $h(r)$ that $M \rightarrow .8928$. This corresponds to $Q^{2} / M^{2}=1.2545$ which is in agreement with the statement that this ratio varies from one for large black holes to two for small holes.

Fig. 15: The radius of spheres in the string metric as a function of the proper distance $\rho$ for a black hole with $r_{0}=10^{-2}$ and $Q=10^{-2}$. The vertical line is the horizon at $\rho=1.08102$.

We conclude this section by demonstrating the formation of a long throat outside the event horizon of a nearly extremal black hole. The geodesic distance $\rho$ from a point $r_{1}$ to 
Fig. 16: The radius of spheres in the string metric as a function of the proper distance $\rho$ for a black hole with $r_{0}=10^{-5}$ and $Q=10^{-2}$. The vertical line is the horizon at $\rho=1.27191$.

a point $r_{2}$ in the string metric is

$$
\rho=\int_{r_{1}}^{r_{2}} \frac{e^{\phi}}{\sqrt{h}} d r .
$$

We will arbitrarily choose $r_{2}=1$, and show in fig. 15 and fig. 16 the radius of the spheres in the string metric $r e^{\phi}$ as a function of the geodesic distance $\rho$ for two different black holes. The black hole in fig. 15 has $r_{0}=10^{-2}$ and $Q=10^{-2}$, so it is not yet near its extremal limit. The mass of this solution is $M=.0086768$. Notice that $r e^{\phi}$ is basically linear until just before the horizon at $\rho=1.08102$. Since $\rho$ measures proper distance inward from a fixed radius outside the horizon, this plot is reversed compared to some of the earlier ones. Increasing $\rho$ corresponds to decreasing radius.

In fig. 16, we show a black hole much closer to its extremal limit, with $r_{0}=10^{-5}$ and $Q=10^{-2}$, which correspond to a mass of $M=.007096$. In terms of proper distance, the horizon is at $\rho=1.27191$. For this black hole, $r e^{\phi}$ levels off before the event horizon. Thus the horizon is down a throat of essentially constant radius. The length of the throat grows as the black hole approaches its extremal limit. Note that the plots fig. 15 and fig. 16 are virtually identical except that the horizon is moved farther down the throat.

\section{Conclusions}

We have studied the behavior of black holes coupled to a massive dilaton. Although exact solutions are not known in closed form, we have obtained a fairly complete picture 
of their properties by combining analytic arguments with numerical integration. One of our main results is that even with a mass, the dilaton can have an important effect in the late stages of black hole evaporation. For black holes with $0<|Q m|<e / 2$, the spacetime initially (when the mass is large) is close to the Reissner-Nordström solution. As the mass decreases, the spacetime becomes closer to the massless dilaton solution. So all of the unusual features of the extremal $m=0$ black holes should emerge in the late stages of the evaporation. The transition occurs when the curvature is still small compared to the Planck scale so higher order string corrections should be negligible. If $|Q m|>e / 2$ and $M \gg Q$, then the black hole solutions have only one horizon. But as the mass decreases, there comes a point where an inner horizon appears. Further evaporation will then be similar to Reissner-Nordström with the extremal limit containing a double horizon.

We have considered only the simplest potential for the dilaton $m^{2} \phi^{2}$. Even though the dilaton is becoming large, this potential becomes negligible near small black holes since other terms in the field equations diverge more quickly. This shows that any potential which grows more slowly than $m^{2} \phi^{2}$ for large $\phi$ should have black hole solutions with similar behavior. (The limit on the charge $|Q m|>e / 2$ for the existence of a double horizon will presumably be modified.) Although the exact dilaton potential in string theory is not known, there have been proposals based on gluino condensation [15]. These potentials typically approach a constant for large $|\phi|$ and so the black holes should be similar to the case we have analyzed. Also, these potentials are typically not symmetric under $\phi \rightarrow-\phi$. This implies that electric and magnetic charged black holes will no longer be related by a duality symmetry.

We have shown that nearly extremal black holes coupled to a dilaton are repulsive. Roughly speaking, this is because the presence of the dilaton near the horizon allows $Q^{2}>M^{2}$, but the dilaton mass cuts off the attractive dilaton force at large distances. This result depends only on properties of the dilaton potential near its minimum, and will hold for any potential that gives the dilaton a mass. We also argued that one consequence of this is that large extremal black holes are quantum mechanically unstable to bifurcation. Since charge is quantized, the extremal black hole of smallest charge must be stable. It will repel black holes with the same charge and attract those with opposite charge. It is interesting to compare this to an elementary particle. The standard argument that the electron cannot be a black hole is that its charge is much larger than its mass. So according

5 In this expression, $e$ is the base of the natural logarithm and not the charge on the electron! 
to the Reissner-Nordström solution, it would have to be a naked singularity. However, we have seen that this is not the case when massive dilatons are taken into account. (Other similarities between black holes with dilatons and elementary particles have recently been discussed in [16].) Could all elementary particles be black holes? One obvious objection is that we have found that black holes can have their charge slightly larger than their mass, while the ratio of the charge to mass of an electron is about $10^{21}$. However we have only considered the case where the dilaton vanishes at infinity. For the magnetically charged black holes that we have been discussing, if $\phi \rightarrow \phi_{\infty}$ asymptotically, the maximal charge to mass ratio is increased by $e^{\phi_{\infty}}$. For electrically charged black holes, the sign of $\phi$ is reversed. So if $\phi_{\infty}$ is negative at infinity, corresponding to weak coupling, the charge to mass ratio can be much larger than one. Another obvious objection is that elementary particles with the same charge can have different masses. Perhaps this could be accounted for when the other interactions are included. Even if the known elementary particles are not small black holes, it is certainly intriguing that in many respects, they act qualitatively the same.

We have also shown that the string metric describing black holes with $M m \approx 1$ can have wormholes outside the event horizon. It is not clear how sensitive these are to the details of the dilaton potential. However it should be kept in mind that we have discussed only classical solutions. These wormholes only occur when $\phi \approx 1$, which is where string loop corrections may become important.

Note added

As this paper was being completed, we received a preprint from R. Gregory and J. Harvey, "Black Holes with a Massive Dilaton," hep-th/9209070, which overlaps with some of this work.

Acknowledgements

It is a pleasure to thank P. Bizon, S. Giddings, R. Gregory, J. Harvey, and A. Strominger for discussions. We also wish to thank the Aspen Center for Physics. This work was supported in part by NSF Grant PHY-9008502 and by DOE grant DE-AC02-76ER03075. 


\section{References}

[1] For review of these puzzles and recent attempts to resolve them, see J. Harvey and A. Strominger, "Quantum Aspects of Black Holes," EFI-92-41, hep-th/9209055.

[2] E. Witten, Phys. Rev. D44, 314 (1991).

[3] G. Gibbons, Nucl. Phys. B207, 337 (1982); G. Gibbons and K. Maeda, Nucl. Phys. B298, 741 (1988).

[4] D. Garfinkle, G. Horowitz and A. Strominger, Phys. Rev. D43, 3140 (1991); D45, $3888(\mathbf{E})(1992)$.

[5] T. Banks, A. Dabholkar, M. Douglas, and M. O'Loughlin, Phys. Rev. D45, 3607 (1992); T. Banks and M. O'Loughlin, "Classical and Quantum Production of Cornucopions at Energies Below $10^{18} \mathrm{GeV}$," Rutgers preprint RU-92-14, hep-th/9206055.

[6] C. Will, Theory and Experiment in Grvitational Physics, Cambridge University Press (1981).

[7] R. Arnowitt, S. Deser and C. W. Misner, Ann. of Physics 33, 88 (1965).

[8] A. Sen, "Rotating Charged Black Hole Solution in Heterotic String Theory," Tata preprint TIFR/TH/92-20, hep-th/9204046.

[9] S. Hawking, Phys. Rev. Lett. 26, 1344 (1971).

[10] D. Brill, Phys. Rev. D46, 1560 (1992).

[11] R. Kallosh, A. Linde, T. Ortin, A. Peet, and A. Van Proeyen, "Supersymmetry as a Cosmic Censor," Stanford preprint SU-ITP-92-13.

[12] M. McGuigan, C. Nappi, and S. Yost, Nucl. Phys. B375, 421 (1992).

[13] P. Bizon and T. Chmaj, "Gravitating Skyrmions," Universitat Wien UWThPh-199223, May, 1992.

[14] A. Hindmarsh, "Odepack," in Scientific Computing, R. Stepleman et al. eds. (NorthHolland, Amsterdam, 1983), 55; L. Petzold, Siam J. Sci. Stat. Comput. 4, 136 (1983). The software is publically available at netlib@research.att.com.

[15] M. Dine, R. Rohm, N. Seiberg and E. Witten, Phys. Lett. 156B, 55 (1986); L. Dixon, "Supersymmetry Breaking in String Theory," in the proceedings of the A.P.S. Division of Particle and Fields Meeting Houston, TX 1990.

[16] C. Holzhey and F. Wilczek, Nucl. Phys. B380, 447 (1992). 